\title{
The amazing graphene: an educational bridge connecting different physics concepts
}

\section{Dominique Persano Adorno ${ }^{\circ}$, Leonardo Bellomonte and Nicola Pizzolato}

Physics Education Research Group, Dipartimento di Fisica e Chimica, Università di Palermo, Italy

E-mail: dominique.persanoadorno@unipa.it

Received 30 April 2017, revised 4 October 2017

Accepted for publication 6 October 2017

Published 18 December 2017

\begin{abstract}
The purpose of this work is to present a learning workshop covering various physics concepts aimed at strengthening physics/engineering student understanding about the remarkable properties of two dimensional materials, graphene in particular. At the basis of this learning experience is the idea of blending and interconnecting separate pieces of knowledge already acquired by undergraduates in different courses and to help them visualize and link the concepts lying beyond separate chunks of information or equations. Graphene represents an appropriate unifying framework to achieve this task in view of its monatomic structure and various exotic processes peculiar to this and some other two dimensional crystals. We first discuss essential elements of group theory and their application to the symmetry properties of graphene with the aim of presenting to physics/electronic engineering undergraduates that in a system characterized by symmetry properties such as a crystal, the acquisition of the solutions of the Schrödinger equation is simpler and easier to visualize than when these properties are ignored. We have then selected and discussed some remarkable properties of graphene: the linear electron energy-momentum dispersion relation in proximity of some edge points of the Brillouin zone; the consequential massless Dirac behaviour of the electrons; their tunnelling behaviour and the related Klein paradox; the chiral behaviour of electrons and holes; the fractional quantum Hall effect in massless particles; and the quantum behaviour of correlated quasiparticles observable at macroscopic level. These arguments are presented in a context covering related pieces of knowledge about classical, quantum and relativistic mechanics. Finally, we mention current applications and possible future ones with the aim of providing students with an expertise that could be useful for further work
\end{abstract}


experiences and scientific investigations regarding new materials, having farreaching implications in various fields such as basic physics, materials science and engineering applications.

Keywords: graphene, physics education, Hall effect, group theory

(Some figures may appear in colour only in the online journal)

\section{Introduction}

The electronic industry has been constrained by the need for making faster and smaller devices characterized by lower and lower power consumption [1]. Conventional technologies based on silicon/germanium seem to be close to their limits. An alternative way, in continuous progress, concerns the synthesis and use of two dimensional (2D) ${ }^{1}$ materials whose groundwork is rather complex and recent, since they were previously considered to be not stable for reasons connected with long range fluctuations, present in large structures of this type, that require little energy to produce breaking of the structure [2]. Although in these structures, both each atom and the entire lattice as a whole are subject to interactions with the environment, and technological difficulties are relevant, different 2D materials consisting of monatomic or diatomic components have become available in the last 10 years. Those of the first type are monolayer or bilayer structures that can be planar or corrugated, those of the second type are almost always bilayer. They may be insulators, semimetals, semiconductors, superconductors or metals [3].

Reduction of dimensionality produces a variety of new effects of interest to research as well as industry, not found in 3D materials. Some of them are: high electronic mobility (appreciably greater than in conventional semiconductors); absence of gap (in monolayer structures and in some bilayer ones) as well as gap presence (mainly in asymmetric bilayer structures), both affect the electron features in a variety of modes; massless behaviour of Dirac electrons that determines tunnelling peculiarities quite different from those usually found in particles subject to quantum mechanics laws; unexpected microscopic collective properties detectable at the macroscopic level (appreciably different from those found in superconductivity or superfluidity); chiral behaviour of electrons and holes (not found in electrons and/or holes in conventional semiconductors); anomalous magnetic properties (the fractional Hall effect) and so on. Well known $2 \mathrm{D}$ compounds are: graphene, silicene, germanene, stanene, boron nitride (BN), $\mathrm{MoS}_{2}$, gallium sulphide (GaS), etc [3]. Graphene is a single layer of graphite, obtained by mechanical exfoliation of small mesas of highly oriented pyrolytic graphite by Novoselov et al in 2004 [4]. Since then, there has been a massive explosion of interest in the study of its properties [5-8]. Indeed, thanks to its remarkable unusual electrical, magnetic and optical properties, the scientific and technological applications of this material seem to be unlimited [9]. Graphene has been and is the object of interesting studies (both theoretical and experimental) and findings since a large number of parameters characterizing this material - such as mechanical stiffness, strength and elasticity, electrical and thermal conductivity, very high electron mobility at room temperature, optical absorption, complete absence of permeability to gases, ability to sustain extremely high densities of electric current-are extraordinary and exceed those characteristic of other materials [10]. All these supreme properties justify its nickname of a 'miracle material'. In view of these peculiar properties, the European GRAPHENE Project was launched in October 2013 [11] with

1 Research about 2D materials was carried out in the recent past in other fields such as high temperature superconductivity in layered materials. 
the aim of building a multidisciplinary research base on this material including electronics, spintronics, photonics, mechanics, etc. Graphene properties are also useful to understand those of other carbon-based materials, including graphite, large fullerenes, nanotubes, etc.

Nowadays, a deeper understanding of the peculiarities characterizing the electron behaviour in graphene is essential in undergraduate education of electronic engineering and physics students, as well as in materials technology, since the current scientific awareness of the quantum peculiarities of graphene is beyond the scope of usual undergraduate courses and outside the experience of most non-specialist physicists. Traditional courses about quantum physics, solid state physics and materials science provide students with a theoretical background on the symmetry properties, free electron and band approximations, relativistic behaviour of the particles, peculiarities of semiconductors, features of 2D materials, etc; however, these courses do not provide students with a sufficient mastery, since the concepts are presented as separate pieces of knowledge, not interconnected. The students, consequently, have difficulties in visualizing and connecting the physics beyond different pieces of information or equations [12, 13]. An efficient instruction method should have a general character, engaging them toward a full comprehension of fundamental concepts and training them to connect transversal facts that at first look may appear to be unrelated [14]. Graphene is a suitable topic to achieve this task.

In the first part of our proposed learning path, we summarize and apply some concepts of solid state physics, such as the symmetry properties of the hexagonal crystal lattice [15], group theory [16] and the calculation of energy states [17, 18]. In fact, the knowledge and application of symmetry operations, peculiar of a periodic structure, allow us to generate a set of wave functions having symmetry properties that greatly help us to visualize the electron state peculiarities and to calculate the energy eigenvalues [17-19]. This phase of the didactic path, designed as an addition to advanced courses in quantum mechanics, could perform several tasks: (i) from an educational point of view, it provides an illustrative example of the advantage of using the concepts of reciprocal space and symmetry; (ii) from a general point of view, it allows the students to achieve a basic awareness of group theory and its use in calculating the electron properties, that could be useful for further work experiences on the structure of other materials; (iii) from a particular point of view, it should provide them with a mastery to deal with the peculiarities of this crystal and of other similar layered hexagonal materials, normally not addressed in standard courses.

The aim of the second part of the learning path is to elucidate some electronic peculiarities of graphene that produce very unusual phenomena, like the Dirac fermions, Klein paradox, chirality and anomalous quantum Hall effect, and their possible technological applications. The proposed activity could be carried out at the end of the regular lectures, in order to avoid interference with the regular classes and to integrate student learning. The learning path could be structured as a five-week workshop with three hours of lectures per week plus two hours of tutorials. An essay should be assigned each week to the class to be due before the next lecture. We believe that the reasoning sequence proposed in this study may help physics/engineering students to surmount eventual epistemological problems and to achieve a mastery of the physics concepts introduced here. Students working in groups should be guided to solve problems, explore models and be engaged in classroom discussions about the problem under study, by assembling links between evidence and explanations. Moreover, such an experience may also help learners to develop the required skills necessary to deal with the inevitable hurdles that are encountered when addressing a research problem [14]. 
The paper is organized as follows. In section 2, we briefly introduce the crystal structure, electronic configuration and symmetry properties of the hexagonal lattice, often overlooked in the textbooks, and summarize in appendix A some basic definitions of group theory. These are applied to the construction of the symmetry dependent wave functions (some examples are reported in appendix B) and to the calculations of the energy levels in the simplified model of the free electrons. In section 3, we will also deal with the effects of the lattice periodic potential on the electron energies (band theory). Special attention is paid to the K-points in the Brillouin zone. Section 4 includes (i) a discussion of the behaviour of the quasiparticles as Dirac fermions with effective massless properties, as a consequence of the linear dispersion in a limited region of the Brillouin zone close to the K-point; (ii) the Klein paradox and chirality. In section 5, we discuss the Hall effect with a particular emphasis on the anomalous quantum Hall effect. Section 6 summarizes some of the possible technological applications of graphene not possible in semiconductor materials currently used. Educational outcomes and concluding remarks are given in Section 7.

\section{Crystal structure, electronic configuration and symmetry properties of graphene}

\subsection{General considerations}

The structure of graphene monolayer consists of a 2D plane honeycomb lattice of densely packed carbon atoms composed of two interpenetrating triangular sub-lattices A and B (red and blue colours in figure 1(a), respectively); by translating a primitive cell (green parallelogram), that contains two non-equivalent carbon atoms, the actual hexagonal lattice is obtained.

The real lattice, as well as the reciprocal one, are simple hexagons [20], as shown in panels (b) and (c) of figure 1. It is convenient to use expressions depending on the primitive vectors $\mathbf{t}$, in the real space and $\mathbf{b}$ in the reciprocal space labelled by the subscripts 1 and 2 indicating the directions of the primitive axes. Their Cartesian components are [17]

$$
\begin{aligned}
& \boldsymbol{t}_{1}=\left(\frac{a}{2}\right)(\sqrt{3} \boldsymbol{i}-\boldsymbol{j}) \quad \boldsymbol{t}_{2}=a \boldsymbol{j} \\
& \boldsymbol{b}_{1}=\left(\frac{2}{a \sqrt{3}}\right) \boldsymbol{i} \quad \boldsymbol{b}_{2}=\frac{1}{a \sqrt{3}}(\boldsymbol{i}+\sqrt{3} \boldsymbol{j})
\end{aligned}
$$

where $a$ is the carbon-carbon distance equal to $1.42 \AA$ [21]. The angle between $\mathbf{t}_{1}$ and $\mathbf{t}_{2}$ is $120^{\circ}$, that between $\mathbf{b}_{1}$ and $\mathbf{b}_{2}$ is $60^{\circ}$ (figures $1(\mathrm{~b})$ and (c)).

Figure 1(a) shows that each carbon atom of a sub-lattice is at $1.42 \AA$ from its three neighbours, belonging to the other sub-lattice and shares one $\sigma$ bond with each of them. All carbon orbitals are $\mathrm{sp}_{2}$ hybridized (one $2 \mathrm{~s}$ orbital together with the $2 \mathrm{p}_{x}$ and the $2 \mathrm{p}_{y}$ orbitals generate three $\mathrm{sp}_{2}$ orbitals). All $\mathrm{sp}_{2}$ orbitals form in-plane $\sigma$-bonds with the $\mathrm{sp}_{2}$ orbitals of the neighbouring carbon atoms. The bonding orbital associated with each $\sigma$-bond is occupied by two electrons with opposite spin. This configuration gives rigidity to the structure, but it does not contribute to the conductivity. The remaining electron, in the $2 \mathrm{p}_{z}$ orbital, contributes to the fourth covalent $\pi$-bond, which is oriented in the $z$-direction (perpendicular to the planar structure). Two atoms per cell contribute to this bond. One can visualize the $\pi$ orbital as a pair of symmetric lobes oriented along the $z$-axis and centred on the nucleus. Each atom has one of these $\pi$-bonds, which are then hybridized together to form what are referred to as the $\pi$-band 
(a)

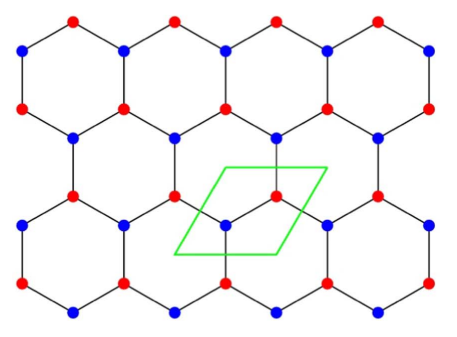

(b)

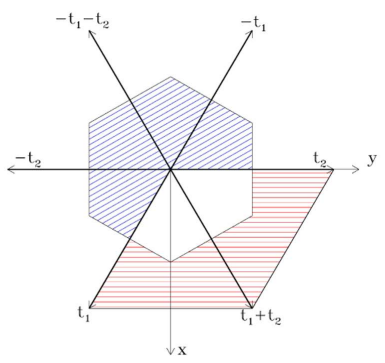

(c)

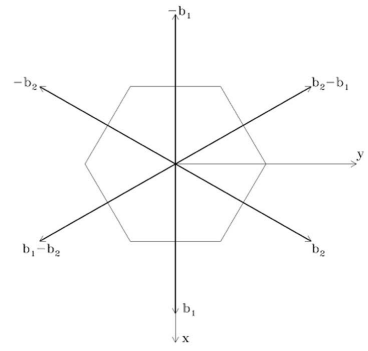

Figure 1. (a) Crystal structure of graphene monolayer; the green parallelogram represents the primitive cell; (b) real lattice and (c) reciprocal lattice.

(a)

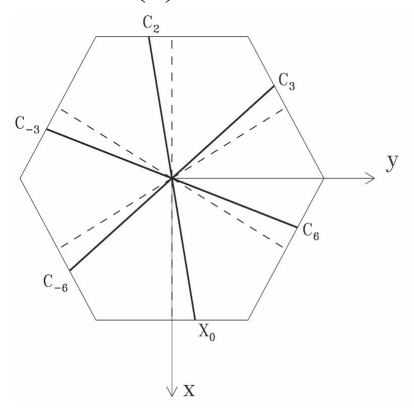

(b)

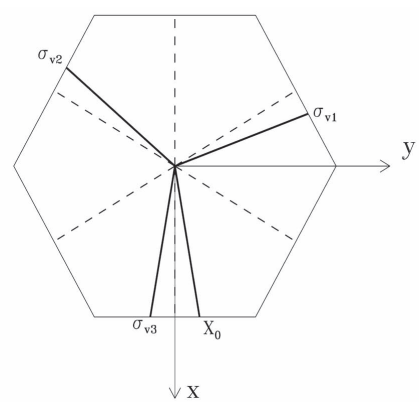

(c)

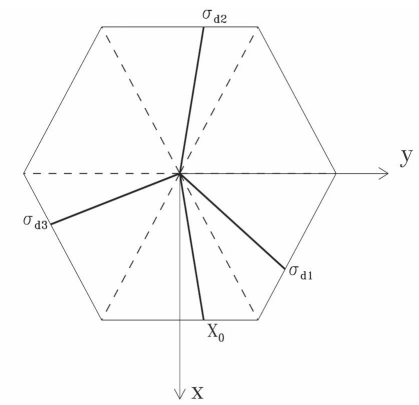

Figure 2. Diagrams illustrating points to which the point marked $X_{0}$ is transformed by the rotations (a), reflections $\sigma_{\mathrm{v}}$ (b) and $\sigma_{\mathrm{d}}$ (c) constituting the group $\mathrm{C}_{6 \mathrm{v}}$.

and $\pi^{*}$-band. These bands are responsible for most of the peculiar electronic properties of graphene.

\subsection{Symmetry properties of the free electron wave functions in the graphene lattice}

The point group of the hexagonal lattice (denoted $\mathrm{D}_{6 \mathrm{~h}}$, see appendix $\mathrm{A}$ for the notation used in group theory) consists of 24 operations. Of these, 12 refer to the rotations about the $z$-axis and reflections about vertical planes. These operations constitute the group $\mathrm{C}_{6 \mathrm{v}}$, subgroup of $\mathrm{D}_{6 \mathrm{~h}}$, and are: the identity (E), five rotations about the $z$-axis: two by $60^{\circ}\left(\mathrm{C}_{6}\right.$ and $\left.\mathrm{C}_{-6}\right)$, two by $120^{\circ}$ $\left(\mathrm{C}_{3}\right.$ and $\left.\mathrm{C}_{-3}\right)$, one by $180^{\circ}\left(\mathrm{C}_{2}\right)$, six reflection vertical planes, three of them bisect two opposite faces $\left(\sigma_{\mathrm{v} 1}, \sigma_{\mathrm{v} 2}\right.$ and $\left.\sigma_{\mathrm{v} 3}\right)$, the others connect two opposite corners $\left(\sigma_{\mathrm{d} 1}, \sigma_{\mathrm{d} 2}\right.$ and $\left.\sigma_{\mathrm{d} 3}\right)$. The panels of figure 2 show the effects of $\mathrm{C}_{6 \mathrm{v}}$ on a generic point $\mathrm{X}_{0}$ [17].

To determine the effects of $\mathrm{C}_{6 \mathrm{~V}}$ on a plane wave, it is convenient to operate in the hexagonal coordinate system. Introducing the dimensionless quantities $\mathrm{h}_{1}, \mathrm{~h}_{2}, \xi, \eta$ peculiar to this system, we have [17]

$$
\psi(\mathbf{r})=\mathrm{e}^{2 \pi \mathrm{i}(\mathbf{k} \cdot \mathbf{r})}=\mathrm{e}^{2 \pi \mathrm{i}\left(\mathrm{h}_{1} \mathbf{b}_{1}+\mathrm{h}_{2} \mathbf{b}_{2}\right) \cdot\left(\xi \mathbf{t}_{1}+\eta \mathbf{t}_{2}\right)}
$$

To visualize the effects of the symmetry operations, we consider, as an example, the rotation of the coordinate system by an angle $+60^{\circ}$. We obtain 
Table 1. Effect of the symmetry operation on a generic coordinate function $\psi\left[\xi \mathbf{t}_{1}+\eta\right.$ $\mathbf{t}_{2}$ ]. The two last columns show how the primitive vectors $\mathbf{t}_{1}$ and $\mathbf{t}_{2}$ transform under the operations of the point group.

\begin{tabular}{llll}
\hline Symmetry operation & Effect of the operation & on $\mathrm{t}_{1}$ & on $_{2}$ \\
\hline $\mathrm{E} \psi[\mathbf{r}]=\psi\left[\xi \mathbf{t}_{1}+\eta \mathbf{t}_{2}\right]$ & Identity & $\mathrm{t}_{1} \rightarrow \mathrm{t}_{1}$ & $\mathrm{t}_{2} \rightarrow \mathrm{t}_{2}$ \\
$\mathrm{C}_{6} \psi[\mathbf{r}]=\psi\left[(\xi-\eta) \mathbf{t}_{1}+\xi \mathbf{t}_{2}\right]$ & Rot $60^{\circ}$ counterclockwise & $\mathrm{t}_{1} \rightarrow \mathrm{t}_{1}+\mathrm{t}_{2}$ & $\mathrm{t}_{2} \rightarrow-\mathrm{t}_{1}$ \\
$\mathrm{C}_{-6} \psi[\mathbf{r}]=\psi\left[\eta \mathbf{t}_{1}+(-\xi+\eta) \mathbf{t}_{2}\right.$ & Rot $60^{\circ}$ clockwise & $\mathrm{t}_{1} \rightarrow-\mathrm{t}_{2}$ & $\mathrm{t}_{2} \rightarrow \mathrm{t}_{1}+\mathrm{t}_{2}$ \\
$\mathrm{C}_{3} \psi[\mathbf{r}]=\psi\left[-\eta \mathbf{t}_{1}+(\xi-\eta) \mathbf{t}_{2}\right]$ & Rot $120^{\circ}$ counterclockwise & $\mathrm{t}_{1} \rightarrow \mathrm{t}_{2}$ & $\mathrm{t}_{2} \rightarrow-\mathrm{t}_{1}-\mathrm{t}_{2}$ \\
$\mathrm{C}_{-3} \psi[\mathbf{r}]=\psi\left[(-\xi+\eta) \mathbf{t}_{1}-\xi \mathbf{t}_{2}\right]$ & Rot $120^{\circ}$ clockwise & $\mathrm{t}_{1} \rightarrow-\mathrm{t}_{1}-\mathrm{t}_{2}$ & $\mathrm{t}_{2} \rightarrow \mathrm{t}_{1}$ \\
$\mathrm{C}_{2} \psi[\mathbf{r}]=\psi\left[-\xi \mathbf{t}_{1}-\eta \mathbf{t}_{2}\right]$ & Rot $180^{\circ}$ & $\mathrm{t}_{1} \rightarrow-\mathrm{t}_{1}$ & $\mathrm{t}_{2} \rightarrow-\mathrm{t}_{2}$ \\
$\sigma_{\mathrm{v} 1} \psi[\mathbf{r}]=\psi\left[\xi \mathbf{t}_{1}+(\xi-\eta) \mathbf{t}_{2}\right]$ & Refl plane vert y $=0(\| \mathrm{x})$ & $\mathrm{t}_{1} \rightarrow \mathrm{t}_{1}+\mathrm{t}_{2}$ & $\mathrm{t}_{2} \rightarrow-\mathrm{t}_{2}$ \\
$\sigma_{\mathrm{d} 2} \psi[\mathbf{r}]=\psi\left[\eta \mathbf{t}_{1}+\xi \mathbf{t}_{2}\right]$ & Refl plane diag $\theta=30^{\circ}$ & $\mathrm{t}_{1} \rightarrow \mathrm{t}_{2}$ & $\mathrm{t}_{2} \rightarrow \mathrm{t}_{1}$ \\
$\sigma_{\mathrm{d}-2} \psi[\mathbf{r}]=\psi\left[(\xi-\eta) \mathbf{t}_{1}-\eta \mathbf{t}_{2}\right]$ & Refl plane diag $\theta=-30^{\circ}$ & $\mathrm{t}_{1} \rightarrow \mathrm{t}_{1}$ & $\mathrm{t}_{2} \rightarrow-\mathrm{t}_{1}-\mathrm{t}_{2}$ \\
$\sigma_{\mathrm{v} 2} \psi[\mathbf{r}]=\psi\left[(-\xi+\eta) \mathbf{t}_{1}+\eta \mathbf{t}_{2}\right]$ & Refl plane vert $\theta=60^{\circ}$ & $\mathrm{t}_{1} \rightarrow-\mathrm{t}_{1}$ & $\mathrm{t}_{2} \rightarrow \mathrm{t}_{1}+\mathrm{t}_{2}$ \\
$\sigma_{\mathrm{v}-2} \psi[\mathbf{r}]=\psi\left[-\eta \mathbf{t}_{1}-\xi \mathbf{t}_{2}\right]$ & Refl plane vert $\theta=-60^{\circ}$ & $\mathrm{t}_{1} \rightarrow-\mathrm{t}_{2}$ & $\mathrm{t}_{2} \rightarrow-\mathrm{t}_{1}$ \\
$\sigma_{\mathrm{d} 1} \psi[\mathbf{r}]=\psi\left[-\xi \mathbf{t}_{1}+(-\xi+\eta) \mathbf{t}_{2}\right]$ & Refl plane diag $\mathrm{x}=0(\| \mathrm{y})$ & $\mathrm{t}_{1} \rightarrow-\mathrm{t}_{1}-\mathrm{t}_{2}$ & $\mathrm{t}_{2} \rightarrow \mathrm{t}_{2}$ \\
\hline
\end{tabular}

$$
C_{6} \psi(\mathbf{r})=\mathrm{e}^{\left.2 \pi \mathrm{i}\left\{\left(\mathrm{h}_{1} \mathbf{b}_{1}+\mathrm{h}_{2} \mathbf{b}_{2}\right) \cdot\left[(\xi-\eta) \mathbf{t}_{1}+\xi \mathbf{t}_{2}\right)\right]\right\}}=\mathrm{e}^{2 \pi \mathrm{i}\left[\left(\mathrm{h}_{1}+\mathrm{h}_{2}\right) \cdot \xi-\mathrm{h}_{1} \eta\right]}
$$

where the last term has been obtained by taking into account the relations between the real and reciprocal lattice; the factor $\left[(\xi-\eta) \mathbf{t}_{1}+\xi \mathbf{t}_{2}\right]$ is a consequence of the effects of this rotation on the coordinates that are $\mathrm{t}_{1} \rightarrow \mathrm{t}_{1}+\mathrm{t}_{2} \mathrm{t}_{2} \rightarrow-\mathrm{t}_{1}$.

Table 1 shows, in analytic format, how a generic function of the coordinates $\psi\left[\xi \mathbf{t}_{1}+\eta \mathbf{t}_{2}\right]$ transforms.

The remaining 12 operations are obtained from the former operations through reflection about the horizontal plane $\left(\sigma_{\mathrm{h}}\right)$ that changes the $\mathbf{z}$-component of an orbital into $-\mathbf{z}$. In the present simplified analysis, based on the free electrons model in a planar structure, the symmetry properties of the wave function of a free electron moving in the $x y$-plane are analyzed using $\mathrm{C}_{6 \mathrm{v}}$. When complete band theory calculations are carried out, the symmetry properties discussed here are still valid but the group $\mathrm{D}_{6 \mathrm{~h}}$ must be used and the Hamiltonian as well as the wave functions are formally more intricate since they involve $\mathrm{s}, \mathrm{p}_{x}$ and $\mathrm{p}_{y}$ orbitals ( $\sigma$-bonds even with respect to $\sigma_{\mathrm{h}}$ ) and $\mathrm{p}_{z}$ orbitals ( $\pi$-bonds odd with respect to $\sigma_{\mathrm{h}}$ ).

\section{Lower electron energy states for monolayer graphene}

\subsection{Free electron model}

The symmetry operations of a lattice leave its Hamiltonian unchanged since it reflects the symmetry of the system. In view of this property and of the orthogonal condition among bases of different irreducible representations, the Hamiltonian has diagonal matrix elements between bases belonging to the same irreducible representation. It follows that when we factorize the secular determinant into blocks of lower order, we shall deal with differential equations whose degree is smaller than that encountered in the case in which the symmetry properties are ignored. If a representation has dimensionality greater than one, we will have a number of degenerate states equal to its dimensionality and their eigenfunctions will behave as bases for that irreducible representation [17]. The knowledge of the energy states allows us to locate eventual degenerations, in the proximity of them there may be an accumulation of energy states available to the charge carriers. 


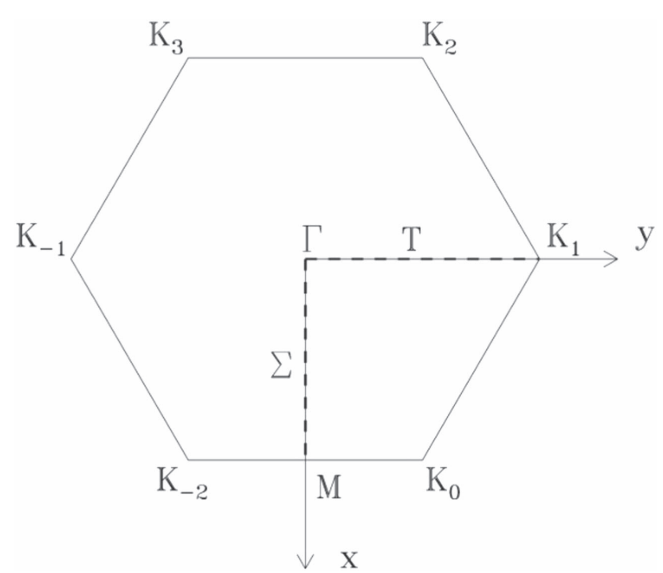

Figure 3. High symmetry points and lines in the graphene reciprocal lattice.

In the free electrons model the electrons are not bound to any particular atom, but the effects of the symmetry related to the presence of the periodicity are accounted for.

The electron wave function $\psi_{\mathbf{K}}(\mathbf{r})$ is characterized by a wave vector $\mathbf{K}$, whose components are along the directions of $\mathbf{b}_{\mathbf{1}}$ and $\mathbf{b}_{\mathbf{2}}$. Since the range of variability of $\mathbf{K}$ is very large and the reciprocal lattice is periodic, it is customary to consider the sum $\mathbf{K}=\mathbf{k}+\mathbf{h}$, where $\mathbf{k}$ may vary inside the first Brillouin zone (reduced wave vector), whereas $\mathbf{h}$ is a reciprocal lattice vector. They are defined as follows:

$$
\boldsymbol{k}=\left(\boldsymbol{k}_{1}+\boldsymbol{k}_{2}\right), \quad \boldsymbol{h}=\left(\boldsymbol{h}_{1}+\boldsymbol{h}_{2}\right)=\left(h_{1} \boldsymbol{b}_{1}+h_{2} \boldsymbol{b}_{2}\right)
$$

with $h_{1}$ and $h_{2}$ integers; the subscripts 1 and 2 indicate the components of $\mathbf{k}$ and $\mathbf{h}$ along the directions of the primitive axes. The Hamiltonian eigenvalues are

$$
\varepsilon_{K}=\frac{\hbar^{2}}{2 m} K^{2}=\frac{\hbar^{2}}{2 m} \cdot\left[\left(\boldsymbol{h}_{1}+\boldsymbol{k}_{1}\right)+\left(\boldsymbol{h}_{2}+\boldsymbol{k}_{2}\right)\right]^{2}
$$

The application of the $h$ operations of the point group on $\mathbf{K}$ will change its direction, leaving its modulus unchanged. The set of these $h$ values is called star of $\mathbf{K}$. However, some particular cases of relevant importance may occur: (i) $\mathbf{K}$ lies along a symmetry direction, (ii) $\mathbf{K}$ touches an edge of the Brillouin zone, (iii) $\mathbf{K}$ terminates at a generic point. The first two cases, denoted as special points, are of interest since the number of different values of $\mathbf{K}$ in the $\operatorname{star}\left(h^{\prime}\right)$ is smaller than $h$. These vectors form a new group (g), that is a subgroup of G. The energies of the vectors obtained upon application of the symmetry operations are equal since they depend on $\mathrm{K}^{2}$.

In the calculation of the electron energy states and degenerations, it is convenient to pay attention to some high symmetry points and/or lines of relevant importance. They are (see figure 3):

- the centre of the Brillouin zone ( $\Gamma$-point);

- the centres of the face of the hexagon $\left(\mathrm{M}_{j}\right.$-points);

- the lines $\Gamma-\mathrm{M}_{j}$ (lines $\sum_{\mathrm{j}}$ );

- the verticess of the face $\left(\mathrm{K}_{j}\right.$ points);

- the lines $\Gamma-\mathrm{K}_{j}$ (lines $\mathrm{T}_{j}$ ).

In order to calculate the wave functions, related eigenvalues and degeneracies, the procedure is 
(1) to choose the line or point of interest and determine the eventual subgroup of the crystal full point group;

(2) to determine the range of variability of $\mathbf{k}$ or its value if a line or a point are considered;

(3) to work out the explicit wave function $\psi_{\mathbf{k h}}(\mathbf{r})$;

(4) to apply to $\psi_{\mathbf{k h}}(\mathbf{r})$ the projection operator defined in appendix A.

Some useful examples of wave function calculations are reported in appendix B.

\subsection{Effects of the periodic potential}

In a periodic structure, a Bloch wave function is

$$
\psi_{\mathbf{K}}(\mathbf{r})=\varphi_{\mathbf{K}}(\mathrm{r}) \mathrm{e}^{\mathrm{i} \mathbf{K} \cdot \mathbf{r}},
$$

where $\varphi_{\mathbf{K}}(\mathrm{r})$ has the periodicity of the lattice and $\mathbf{K}$ is the wave vector. It describes a oneelectron state (or two if spin is considered) and has the same amplitude at equivalent positions in the lattice.

A translation of a quantity $T_{n}=n r_{j}$ adds to the wave function a phase factor according to

$$
\psi_{\mathbf{K}}\left(\mathbf{r}+\mathbf{T}_{\mathbf{n}}\right)=\psi_{\mathbf{K}}(\mathbf{r}) \mathrm{e}^{\mathrm{i} \mathbf{K} \cdot \mathbf{T}_{\mathbf{n}}}
$$

If the potential energy is null, constant or weak with respect to the kinetic energy of the electrons being considered, the free electron approximation applies since $\varphi_{\mathbf{K}}(\mathrm{r})$ reduces to a normalization constant. When we are dealing with bound electrons (the valence states, $\sigma$ bonds in graphene), $\varphi_{\mathbf{k}}(\mathrm{r})$ will be appreciably varying in the neighbourhood of the nuclei where it tends to approximate the wave function of an inner shell electron of the free atom, whereas it tends to become a smooth function in the intermediate regions.

A method used in calculations that take into account the presence of the crystal potential in the Hamiltonian is the tight binding method. We give a short summary of it [22, 23]. Consider two atoms each with a valence electron in the outer shell. When they are brought together, their wave functions $\psi_{1}$ and $\psi_{2}$ partially overlap. The possible combinations are: $\psi_{1} \pm \psi_{2}$. These new states are characterized by different energies since the charge distributions in the space in between the two nuclei are different. Consequently, a twofold degenerate state corresponding to the two atoms far apart splits into two non-degenerate states. This argument can be extended to a long chain of atoms; the periodic perturbation due to the crystal potential removes almost completely the degeneration and the N-degenerate states are replaced by $\mathrm{N}$ levels constituting a bunch. The new wave functions are Bloch functions and are used in the calculation of the electron energies by diagonalizing the Hamiltonian. It follows that the crystallographic potential determines rearrangements of the dispersion curves both in value and shape with respect to those found in the free electron model. A pioneering work about disclosing the band features of the graphite lattice has been carried out by Wallace [21] in 1947, using the tight binding approximation. Other relevant papers are those by Lomer [24], Malard et al [7] and the recent and exhaustive papers by Kogan et al [8, 25, 26]. These authors have prevalently used the tight binding method, occasionally the OPW-pseudopotential approximation [23].

The panels of figure 4 report the dispersion curves in the reduced Brillouin zone, calculated according to the free electron model (left panel) and to the tight binding model (right panel). It must be taken into account that in the free electron model the energy is always positive, whereas in band energy calculations the zero energy is taken at the Fermi level. For the sake of comparison between the two panels, we have shifted the energy scale in the left panel, setting the zero value at the same value of the right panel. 

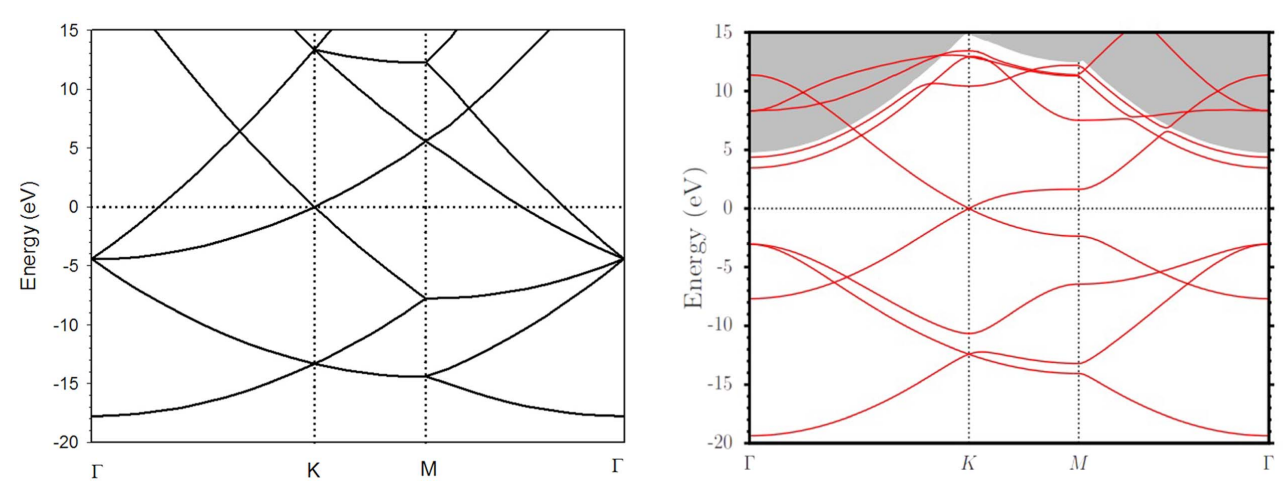

Figure 4. Left: Lowest energy values in the hexagonal lattice along the symmetry lines $\mathrm{T}(\Gamma-\mathrm{K}), \mathrm{T}^{\prime}(\mathrm{K}-\mathrm{M}), \sum(\mathrm{M}-\Gamma)$ and at their edge points, calculated by ignoring the crystal potential. Right: Band structure evaluated with the use of the full potential linearized plane wave method (FP-LAPW). The red-marked lines are well converged single graphene layer bands, while the grey background corresponds to a continuous spectrum. Figure adapted with permission from Ref. [8]. Copyrighted by the American Physical Society.

The parabolic shape of the dispersion curves in the free electron model becomes approximately parabolic in the tight binding model; the values of $\varepsilon(\mathbf{k})$ consequently differentiate and some degenerations typical of the free electron approximation are removed. A remarkable result is the unusual behaviour near the K-points at zero energy in the right panel of figure 4 . The valence band and the conduction band touch each other (absence of gap) and the $E-k$ curve becomes linear with good approximation. Due to this linear behaviour, both electrons and holes behave as relativistic fermions obeying the Dirac equation, discussed in section 4.1. Consequently, the six K-points are denoted Dirac points. They are the principal points of interest when studying the electronic properties of graphene. This is noteworthy in comparison to conventional semiconductors where the primary point of interest is generally $\Gamma$, where momentum is zero [27].

This linear behaviour, noted by Wallace [21], has been explained by Katsnelson et al [28] as a consequence of the graphene crystal structure consisting of two non-equivalent carbon lattices. Quantum mechanical hopping between the two lattices determines the formation of two bands that intersect at the edge of the Brillouin zone at the K-point where the dispersion is linear and the particles exhibit a massless behaviour. The Fermi level crosses the energy scale at this K-point [6]. Both electrons and holes in the upper band and in the lower one, respectively, contribute to the electric conductivity [21].

At the end of this part of the workshop our students should have acquired a necessary knowledge of the effects of the crystal potential useful to treat the problem of calculating the energy levels in the context of band theory. This knowledge/expertise could be useful for further work experiences on other crystalline materials.

\section{Massless fermions, Dirac equation and the Klein paradox}

\subsection{Dirac equation}

As mentioned above, the dispersion curve, obtained by expanding the full band structure in proximity of the K-points as $\mathbf{k}=\mathbf{K}+\mathbf{q}$, with $|\mathbf{q}| \ll|\mathbf{K}|$, has an approximately linear shape [21]: 


$$
E_{ \pm}(\boldsymbol{q})=\hbar v_{F}|\boldsymbol{q}|+O\left[\left(\frac{q}{K}\right)^{2}\right]
$$

where $\boldsymbol{q}$ is the momentum measured relatively to a K-point, $v_{F}$ is the Fermi velocity, $\left(v_{F} \approx 10^{6} \mathrm{~m} \mathrm{~s}^{-1}\right.$, or $1 / 300$ th the speed of light in vacuum [27]) and zero energy has been taken at the K-point [8].

It follows that the dispersion relation depends only on the magnitude of the wave vector and represents a set of modes forming the surface of a pair of cones in the energy-momentum space that touch at the K-point in monolayer graphene. The upper cone represents modes with positive energy with respect to the K-point energy, the lower one represents negative energy modes. In this approximation, there is no gap and these quasiparticles (Dirac fermions) behave differently from the charge carriers in metals or semiconductors, where the energy spectrum has an approximate parabolic dispersion relation.

To deepen this aspect it is worth recalling and comparing the equations governing a quantum free particle with those to be used for a relativistic one.

The Schrödinger equation is homogenous and linear in order to respect the property of superposition, and it is a differential equation of first order with respect to time specifying that the solution $\psi(\mathrm{r}, \mathrm{t})$ at a given initial state uniquely defines the time evolution of the system. This equation does not satisfy the principle of relativity that treats space and time as a whole. In the relativistic context both space and time derivatives should enter symmetrically in the differential equation and must be of the same order. The momentum and energy that are derivatives of the space-time vector are related by the relation

$$
E^{2}=\left(p^{2} c^{2}+m^{2} c^{4}\right)=\left(k^{2} \hbar^{2} c^{2}+m^{2} c^{4}\right)
$$

where $m c^{2}$ is the relativistic particle (the electron) rest energy. It follows that

$$
E= \pm \sqrt{\left(p^{2} c^{2}+m^{2} c^{4}\right)}= \pm \sqrt{\left(k^{2} \hbar^{2} c^{2}+m^{2} c^{4}\right)}
$$

which infers positive as well as negative energy values for a free particle. In order to solve this puzzle, Dirac suggested the existence of antiparticles occupying the states with negative energy. If the available states are completely occupied the exclusion principle forbids transitions among them. Vice versa, at $\mathrm{T}>0$, transitions become possible: some electrons occupying the upper states of the lower band move in the upper band and the empty states in the lower band are occupied by holes, their antiparticles.

Proceeding in a similar way to that adopted to obtain the Schrödinger equation, it is possible to formulate the equation

$$
-\hbar^{2} \frac{\partial^{2}}{\partial t^{2}} \psi(\mathrm{r}, \mathrm{t})=-\hbar^{2} c^{2} \nabla^{2} \psi(\mathrm{r}, \mathrm{t})+m^{2} c^{4} \psi(\mathrm{r}, \mathrm{t}),
$$

known as the Klein-Gordon equation, that satisfies the criteria of homogeneity and linearity, but it does not satisfy the criterion of being of first order with respect to time.

\subsection{Klein paradox and chirality}

An interesting feature of relativistic particles is their insensitivity, under some conditions, to external electrostatic potentials, known as the Klein paradox. According to it, the Dirac fermions can be transmitted with probability close to unity through a classically forbidden region. We here give a simplified visualization of the Klein paradox similar to that used by other authors in dealing with graphene [28-30]. An exhaustive account can be found in [31]. The behaviour of a moving particle when it encounters a step potential $V_{0}$, greater than its kinetic energy, depends critically on its nature. A classical particle is reflected; a quantum one 


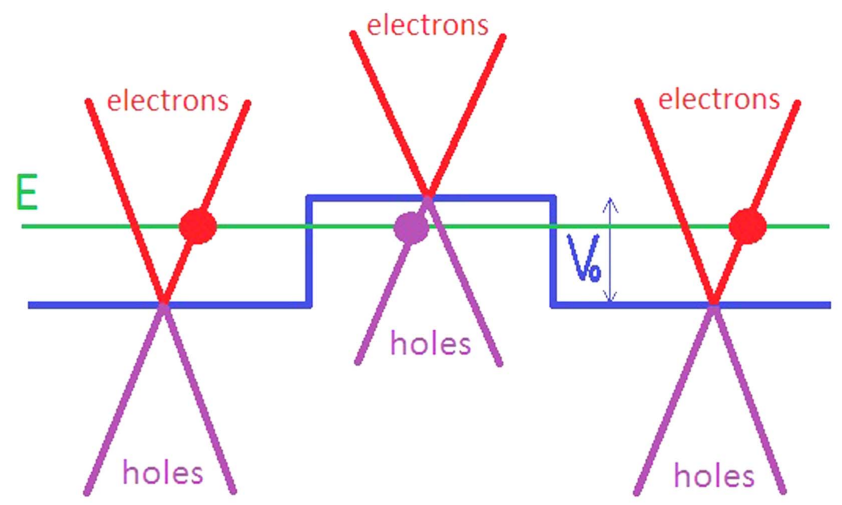

Figure 5. Schematic diagram of the spectrum of quasiparticles in single-layer graphene. The diagrams illustrate the values of the Fermi energy $E$ across the potential barrier of height $V_{0}$.

can tunnel though the barrier although the tunnelling probability decreases exponentially with the thickness and height of the barrier. For relativistic particles, governed by the Dirac equation, the situation is more complex: consider an electron in the upper state of figure 5, travelling in the direction of increasing values of $x$ with a positive value of $k$ in the absence of potential. If the particle encounters a potential step $V_{0}$, one must take into account whether the quantity $E-V_{0}$, is greater than zero or not. In the first case, the electron will continue to propagate with a new value of $k$ satisfying the relation

$$
E-V_{0}=\sqrt{\left(k^{2} \hbar^{2} c^{2}+m^{2} c^{4}\right)}
$$

In the second case, one must distinguish two cases: (i) if $E-V_{0}<m c^{2}$, it will be $k^{2}<0$, the momentum inside the potential barrier is imaginary and the wave function decays exponentially, (ii) if $V_{0}$ is sufficiently high so that $E-V_{0}<-m c^{2}$, it can be $E-$ $V_{0}=-\left[\hbar^{2} \mathrm{k}^{2} c^{2}+m^{2} c^{4}\right]^{1 / 2}$ and again $k^{2}>0$. In this case, the propagation of an electron becomes possible for states in the lower branch since the electron is turned into a hole inside the barrier and changed back to an electron outside the barrier. Therefore the propagation is possible and the transmission probability approaches the value 1 only for low-energy electrons and very high values of the potential barrier $V_{0}$, in stark contrast with nonrelativistic particle tunnelling.

The group velocity $\mathrm{d} k / \mathrm{d} x$ is positive for particles of the upper branch with positive values of $k$ as well as for particles of the lower branch with negative values of $k$. Consequently, an electron continues to propagate with a negative momentum. Two particles, one in the upper band and one in the lower band, behave as an electron and a hole (or a positron in nuclear physics) since they have identical properties, except for their charges that are opposite $[9,28]$. This property is called chiral behaviour ${ }^{2}$. This behaviour does not occur in semiconductors in which the Schrödinger equations as well as their solutions are different for electrons and holes, due to the different effective masses and shapes of the dispersion curves.

At the end of this part of the workshop our students should have gained familiarity with the main peculiarities of the electron behaviour of graphene, consolidating their knowledge

2 An object is said to be chiral if it exists in two forms which are mirror images of one another; the human hands are a simple example of chiral objects, in fact the mirror image of the right hand is the left hand. 
about the equations governing the motion of a classical free particle, a quantum free particle, or a relativistic one.

\section{The Hall effect}

The Hall effect is peculiar of systems with free or nearly free charged particles whose motion is affected by a magnetic field in the presence of an electric field. We here consider 'different' Hall effects, characteristic of $2 \mathrm{D}$ materials:

1. The classical Hall effect (CHE): it occurs at room temperature in the presence of a longitudinal electric field and a transverse weak magnetic field in a conductor;

2. The integer quantum Hall effect (IQHE): it occurs at low temperature in the presence of strong transverse magnetic fields. It cannot be treated as a perturbation since it requires a new quantization rule;

3. The fractional quantum Hall effect (FQHE): it is a particular case of the previous one. It occurs in few 2D materials (graphene is one of them) in which the electrons acquire a collective behaviour and exhibit unexpected features.

\subsection{Classical Hall effect}

Consider a 2D conductor lying in the $x y$-plane, and apply an electric field $\boldsymbol{E}_{\mathbf{x}}$ and a magnetic field $\boldsymbol{B}_{\mathbf{z}}$. The total force $\mathbf{F}$ acting on a charge $-e$ moving with velocity $\mathbf{v}$ is [22]

$$
\mathbf{F}=-\mathrm{e}\left(\mathbf{E}_{\mathbf{x}}+\frac{1}{\mathrm{c}} \mathbf{v} \times \mathbf{B}_{\mathrm{z}}\right)
$$

The two contributions in equation (12) influence the electrons lying in the Fermi sphere by impressing upon them a longitudinal acceleration and a centripetal acceleration. The effect of the random collisions, with average time $\tau$, acts as a frictional damping term in the equation of motion:

$$
\mathbf{F}=\frac{\mathrm{d} \mathbf{p}(t)}{\mathrm{d} t}+\frac{\mathbf{p}}{\tau}=m\left[\frac{\mathrm{d} \mathbf{v}(t)}{\mathrm{d} t}+\frac{\mathbf{v}}{\tau}\right] .
$$

At equilibrium, the induced current $\mathrm{I}$ is constant and the field $E_{y}=-E_{x} \mathrm{e} B_{z} \tau / m c$ (Hall field, whose value is deducible from the above equations subject to the condition $\mathbf{v}=$ const), set up by the electrons that accumulate on one $y$-direction and deplete on the opposite one, prevents further charge accumulation balancing the Lorentz force. This effect is called the Hall effect.

Some other quantities or effects deducible from the above relations, valid at equilibrium $(\mathrm{d} \mathbf{p}(t) / \mathrm{d} t=0)$, will be of central interest in the following discussion. They are:

(i) the cyclotron frequency, $\omega_{\mathrm{c}}=\mathrm{e} B_{z} / m c$. It is the frequency of the rotatory motion impressed in the $x y$-plane by the Lorentz force acting on a moving free charge;

(ii) the Hall coefficient, $\mathrm{R}_{\mathrm{H}}=E_{y} / \mathrm{j}_{x} B_{z}=-1 /$ nec. It is proportional to the Hall field and depends on the sign and value of the surface charge concentration; it is often used to determine both quantities [22];

(iii) the material resistivity or magnetoresistance $\rho_{\mathrm{xx}}$. It is a constant, in fact it only depends on some peculiarities such as the surface charge density;

(iv) the Hall resistivity $\rho_{\mathrm{xy}}$, defined as $V_{\mathrm{H}} / I\left(V_{\mathrm{H}}\right.$ is the Hall voltage along the $y$-side) is proportional to $B_{z}$. 


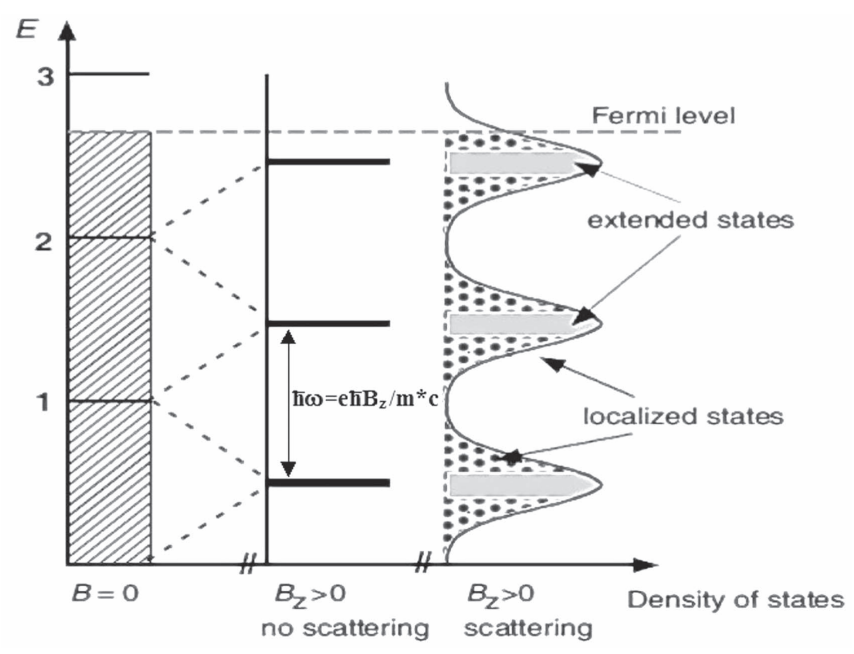

Figure 6. Left: Quasi-continuous states of a Fermi electron gas at $T=0$ in the absence of perturbations. Centre: rearrangement of the electron distribution on the left in the presence of a magnetic field and absence of impurity/phonon scattering (Landau levels). Right: Rearrangement of the electron states in the presence of impurities that cause scattering and electron trapping (localized states). Reproduced with permission from [33].

\subsection{Integer quantum Hall effect}

This effect was discovered by von Klitzing [32] a century after the discovery of the CHE. Before entering into its details, we must point out some peculiarities:

(a) If the product $\omega_{\mathrm{c}} \tau$ is small, the electrons cannot complete an orbit between two collisions. To enhance it, there are two ways that can be added together. One consists of reducing the temperature $T$ dependent processes that are responsible for the electron-phonon collisions: as $T$ is lowered, $\tau$ is enhanced. The other consists in increasing $B_{\mathrm{z}}$ and, consequently, $\omega_{\mathrm{c}}$. Temperatures of the order of $1 \mathrm{~K}$ or less and magnetic fields of 1-10 Tesla are necessary to carry out the experiments discussed below; under these conditions, $\omega_{\mathrm{c}} \tau \gg 1$ and the electrons may complete many orbits without suffering collisions.

(b) Let us consider a gas of independent (free) electrons. As a consequence of their quantum behaviour, in the absence of external fields the quantum numbers $\mathrm{k}_{x}$ and $\mathrm{k}_{y}$ are equally spaced in a square sample (see for example figure 9.24a in [22]) and the energy levels are $\mathrm{E}(\mathrm{k})=\left(h^{2} / 2 m\right)\left(\mathrm{k}_{x}{ }^{2}+\mathrm{k}_{y}{ }^{2}\right)$. The presence of a field $B_{\mathrm{z}}$ produces a rearrangement of both electron motion and related energies. The orbits become circular (see figure $9.24 \mathrm{~b}$ in [22]), $\mathrm{k}_{x}$ and $\mathrm{k}_{y}$ are no longer good quantum numbers and the quasi-continuous quadratic dispersion law is replaced by a discrete one, whose values $E_{n}$, denoted Landau levels, are quantized and proportional to $B_{\mathrm{z}}$ according to

$$
E_{n}=\left(n+\frac{1}{2}\right) \hbar \omega_{\mathrm{c}}, \quad(n=1,2,3 \ldots .) .
$$

The number of allowed orbitals on each orbit is constant for a given value of the magnetic field. The electrons cannot reside in the energy gaps in between or in the quasi-continuous $\mathrm{k}_{z}$ (unaffected by $B_{z}$ ) states that do not exist in a $2 \mathrm{D}$ crystal. Figure 6 shows how the transition 


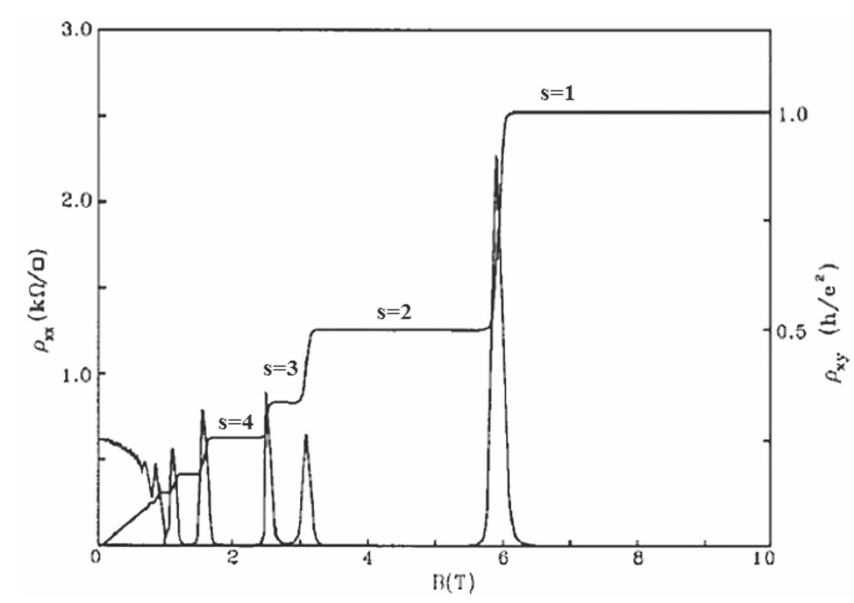

Figure 7. $\rho_{x x}$ and $\rho_{x y}$ of a relatively low-mobility $2 \mathrm{D}$ electron gas in GaAs $/ \mathrm{Al}_{x} \mathrm{Ga}_{1-x} \mathrm{As} ; T=66 \mathrm{mK}, n=1.93 \cdot 10^{11} \mathrm{~cm}^{-3}$. Reproduced with permission from [34]. Copyrighted by the American Physical Society.

from the quasi-continuous Fermi levels to the highly degenerate Landau levels occurs. The degeneration $D$ increases with $B_{\mathrm{z}}$ according to the law $D=\rho \mathrm{B}_{\mathrm{z}}$ with $\rho=\mathrm{e} \mathrm{L}_{x} \mathrm{~L}_{y} / h c$ [22]. The population of the occupied Landau levels consequently depends on $B_{\mathrm{Z}}$ (to be considered as a variable) and on the size of the conductor (a constant). The electrons occupy these levels in increasing order up to the last one that may be completely filled or not. These properties apply to an ideal crystal structure.

In a real lattice, in which defects and impurities are unavoidable, things may be different. Let us suppose that only the lowest Landau level is completely filled and keep reducing $B_{z}$; the degeneration reduces too and an increasing number of electrons is compelled to migrate to the contiguous level that may be too high. From the energetic point of view, it will be more convenient for them to be trapped at localized impurity sites. Such electrons are not mobile and, consequently, they do not participate in the conductivity. A further reduction of $B_{\mathrm{z}}$ reduces the Landau energy separation and it becomes convenient for the trapped electrons to occupy an available lower empty level. It turns out that the Landau levels in a real lattice are completely filled up or completely empty over a wide range of $B_{z}$.

The IQHE shown in figure 7 consists of the concomitant quantization of the Hall resistivity $\rho_{x y}$ characterized by a step behaviour and in the drop of the magnetoresistance $\rho_{x x}$ to very low values [34]. Both are bound to the phenomenon of electron localization-delocalization and deserve a simultaneous discussion. The plateau in each step is explained in the context that there are always filled Landau levels with the exception of those values of $B_{\mathrm{z}}$ falling in the narrow ranges in which a transition from the last occupied Landau level to the next one occurs.

The experiments show that $\rho_{x y}$ varies by steps governed by the law

$$
\rho_{x y}=\frac{\mathrm{h}}{s \mathrm{e}^{2}}
$$

where $s$ is an integer greater than zero and depends on $B_{\mathrm{z}}$. The coefficient $\mathrm{h} / \mathrm{e}^{2}$ is a universal constant independent of the sample employed. It only depends on the value of the electronic charge. 
The magnetoresistance $\rho_{x x}$ is consequently null since when some Landau levels are completely filled and others completely empty, there is no possibility of electron scattering, $\tau$ is extremely large and $\rho_{x x}$ is consequently null. In the narrow transition region in which $s$ assumes the contiguous value there are non-completely filled levels, scattering is possible, consequently $\rho_{x x}$ is not null.

It is worth observing that in an ideal structure, without defects, the IQHE will not be possible.

\subsection{Fractional quantum Hall effect}

This effect was discovered by Tsui et al in 1981 in 2D heterojunctions in which the lowest Landau level is partially occupied because of the low electron density [34]. The FQHE is detectable in these materials at very low temperatures $(<0.5 \mathrm{~K})$ and extremely high magnetic fields $(>150 \mathrm{~T})$. The main difference between the IQHE and the FQHE is that electrons behave as free (non-interacting) particles in IQHE and correlated interacting particles in the second case. The equal spacing of the Landau levels is here replaced by a collective behaviour of composite particles whose charge is smaller than $e$ and the level separation is proportional to $B_{z}{ }^{1 / 2}$. This effect was not expected since the charge of composite particles, such as the Cooper pairs in superconductivity, is a multiple of $e$. It has been explained in the context that bits of magnetic field are attached to each electron creating a new object whose properties are different from those of a free electron [32]. These particles experience an effective magnetic field $B_{\mathrm{z}}{ }^{*}$ that is different from the applied field $B_{z}$. Their motion seems to not depend strictly on the magnetic field and they may behave as bosons as well as fermions depending on the magnetic field. Due to the complexity of the problem, the treatment of the FQHE cannot be as rigorous as that in the IQHE.

The most relevant outcomes can be resumed as follows [35]:

(a) electron condensations occurs at about $1 \mathrm{~K}$ in the presence of magnetic fields of the order of $150 \mathrm{~T}$;

(b) about half of the lowest Landau level is complete;

(c) the electric conductivity is extremely high and the Hall conductance changes from $\mathrm{e}^{2} / \mathrm{h}$ to $\mathrm{e}^{2} / 2 \mathrm{~h}$;

(d) this process is little affected by the presence of small deviations of the electron density;

(e) condensation does not occur in all samples;

(f) particle masses are bound to the interactions with $B_{z}$ and to the particle-particle interactions and can be different from the free electron mass.

The consequence is that strongly interacting fermions in an external magnetic field $B_{z}$ transform into weakly interacting ones in the presence of a residual field $B_{z}{ }^{*}$ smaller than $B_{z}$.

To conclude, both quantum Hall effects are rare examples of microscopic effects on a macroscopic scale and have allowed us to establish very precise values of microscopic quantities such as the electron charge and the Planck constant that can be measured without being disturbed by the influence of the materials being used. Moreover, the fractional Hall effect helps us understand some physics governing interacting particles.

5.3.1. FQHE in massless particles (graphene in particular). Graphene differentiates from heterojunctions for the linear dispersion relation in the proximity of Dirac points [36]. We recall that near these points of the Brillouin zone, electrons exhibit a relativistic massless behaviour characterized by the dispersion law $E(\mathbf{q})= \pm \mathrm{h} v_{F}(\mathbf{q})$. It is obvious to expect the occurrence of processes not possible in the cases in which the usual dispersion law applies 


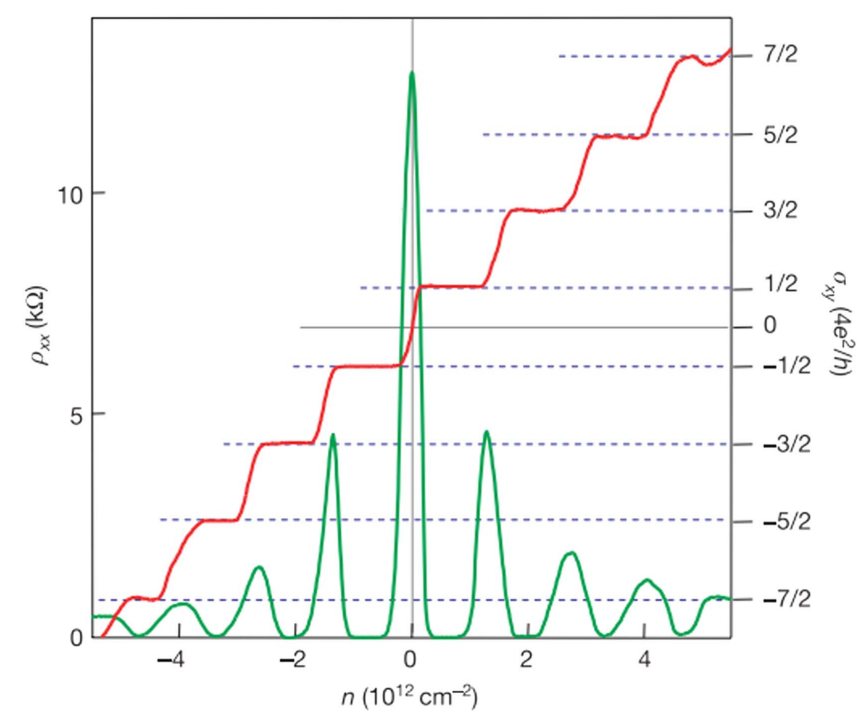

Figure 8. Hall conductivity $\sigma_{x y}$ and longitudinal resistivity $\rho_{x x}$ of graphene as a function of their concentration at $B=14 \mathrm{~T}$ and $T=4 \mathrm{~K}$. Adapted by permission from Macmillan Publishers Ltd: Nature Publishing Group [37], Copyright (2005).

and, in fact, some aspects of the FQHE in graphene differentiate from those previously described.

The FQHE effect in graphene, discovered by Novoselov et al [37], is characterized by the following experimental peculiarities: (i) the conductivity never falls below a given minimum, (ii) the quantum Hall effect occurs at certain half values, (iii) the cyclotron electron mass is no longer their effective mass by the mass of a relativistic particle given by $E=m c^{2}$, and (iv) the Landau energy levels are now proportional to $B^{1 / 2}$.

To discuss these results, we must recall that at the K-points the conduction and valence bands have a conical behaviour. For the energy values, solutions of the relativistic equation are positive for the upper band and negative for the lower one. Their degeneracy obeys the following criterion: let us call $\mathrm{N}_{\mathrm{L}}$ the number of available sites in a level, the degeneracy of the ground state is $\mathrm{N}_{\mathrm{L}}$; it is occupied by $\mathrm{N}_{\mathrm{L}} / 2$ electrons and by an equal number of holes although with opposite chirality. The degeneracy of the other levels is $2 \mathrm{~N}_{\mathrm{L}}$ : they are occupied by $\mathrm{N}_{\mathrm{L}}$ electrons and by $\mathrm{N}_{\mathrm{L}}$ holes [38]. This fact helps us to understand the experimental results found by Novoselov et al reported in figure 8 [37].

The transversal conductance $\sigma_{x y}$ (along the $y$-direction) is quantized in steps with half integer multiples of $4 \mathrm{e}^{2} / \mathrm{h}$ (right hand scale in figure 8) and can assume positive as well as negative values ascribed to the electrons whose energy is greater than zero with respect to the Fermi level or to the hole conductivity whose energy is negative, respectively. The longitudinal resistivity $\rho_{x x}$, whose scale is on the left, is close to zero in each plateau interval.

It is worth comparing figure 7 for the IQHE to figure 8 for the FQHE in graphene. The main difference is bound to the presence of electrons in the first case and to both massless degenerate states of electrons and holes (chiral particles) in the second case. The horizontal and the vertical scales must be different: in figure 7 the results are plotted as a function of $B_{\mathrm{z}}$, in figure 8 the horizontal axis represents the density of charge that is zero at the lowest Landau level (Dirac point), it increases on the right hand side for the electrons and on the left for holes. The plateaus are present in both figures although with different meanings: in 
figure 7 , they indicate when a variation of the magnetic field is capable of producing the occupation of a previously empty Landau level, as stated before, the degeneracy of these levels depends on $B_{\mathrm{z}}$. Vice versa, in the second case the results are plotted as function of the particle density.

The major limit to commercial applications of the quantum Hall effects is inherent to the need for extremely low temperatures (less than $1 \mathrm{~K}$ ) and high magnetic fields. The former is a more relevant limitation than the latter. However, Novoselov et al in 2007 have pioneered research having the aim of avoiding the first difficulty [39]. They were successful in showing that in single-layer graphene the quantum Hall effect can be observed at room temperature. This is possible for various reasons. The most relevant is the unusual nature of the charge carriers in this material, they behave as massless relativistic particles whose motion is little affected by scattering. The large value of the cyclotron frequency $\left(\omega_{\mathrm{c}}\right.$ proportional to $\left.\mathrm{m}^{-1}\right)$, characteristic of these fermions determines an energy gap equivalent to about $3 \cdot 10^{3} \mathrm{~K}$ between the ground state $(\mathrm{N}=0)$ and the first excited state $\mathrm{N}= \pm 1$ that exceeds the thermal energy by a factor of about 10 . Other effects that help the survival of the quantum Hall effect at room temperature are bound to the graphene high carrier concentration, essential for an appreciable population of the lowest Landau level (this does not occur in heterostructures such as GaAs) and to quasi-temperature independent mobility.

At the end of this part of the workshop our students should have consolidated their knowledge about the Hall effect and achieved a more meaningful understanding of relevant concepts regarding the quantum behaviour of correlated particles [40]. In particular they should be able to make an analogy between the Cooper pairs in superconductor materials and the composite electrons in graphene, and appreciate analogies/differences between the concepts of the effective mass of electrons in semiconductors and the effective charge of Dirac fermions in graphene.

\section{Possible technological applications of graphene and other two dimensional structures.}

Graphene is a no gap semimetal characterized by a number of extremely useful properties, including very fast electrical mobility (the speed at which electrons move when a voltage is applied) an order of magnitude higher than that of $\mathrm{Si}$, high transparency, high mechanical strength and excellent thermal conductivity, which make it suitable for building nanoelectromechanical devices. The gapless nature of its band structure makes it capable of absorbing light across a very broad range, from UV to IR. Since each atom of carbon resides on the crystal surface, it offers the largest possible contact area with its environment; this makes it an ideal platform for sensors. A graphene monolayer on a semiconductor constitutes a Schottky junction [41]. Whereas the Fermi level is stable in a metal, it can be adjusted in graphene in various manners: by applying an appropriate bias voltage; by chemically doping the material with impurities; by shining light on the junction. The possibility of manipulating and controlling these external factors makes it a valuable component for optoelectronic devices. As an application, we remark that the graphene based Schottky junctions form the basis for solar cells, with efficiency comparable with that of commercial Si cells, photodetectors, lasers, LEDs, chemical sensors and so on [41].

As we have seen in section 4 , in proximity to the Dirac points the low-energy excitations are massless, chiral, Dirac fermions. However, due to the zero density of states at the Dirac points, electronic conductivity is actually quite low, but it can be increased by doping (with either acceptors or donors) in order to create a material that is potentially a better conductor 
than copper at room temperature. Unlike conventional semiconductors, the absence of an energy band-gap in graphene makes it unsuitable for building digital switches, but very appropriate for very fast analogue electronics like high-frequency transistors or RF mixer circuits.

The unusual behaviour of the Dirac electrons can be controlled by the application of external electric and magnetic fields, or by altering the sample geometry and/or topology. In particular, the tunnelling properties in graphene monolayer, discussed in section 4.2 , are expected to play an important role in its transport properties, especially in the regime of low carrier concentrations where disorder induces significant potential barriers and the system is likely to split into a random distribution of p-n junctions. In fact, in conventional 2D systems, strong enough disorder is the cause of the presence of electronic states separated by barriers whose transparency decreases exponentially [28]. This behaviour is known to conduct to the Anderson localization [42]. In contrast, all potential barriers in graphene are relatively transparent at least for some angles. This fact does not allow the confinement of charge carriers by potential barriers that are smooth on the atomic scale. Therefore, different electron and hole 'puddles' induced by disorder are not isolated but effectively percolate, thereby suppressing localization. In this case, it has been experimentally established that electrons can propagate in graphene without scattering over distances of the order of micrometres [6]. Their chiral nature can be used for applications where one can manipulate the valley index besides its charge $[43,44]$ (valleytronics), in a way similar to the control of the spin in mesoscopic devices (spintronics [45-47]). Very recently, it has been found that thanks to electronelectron scattering in highly pure graphene the electrons collectively flow as a viscous liquid [48, 49].

Graphene can be considered the starting point for all investigations regarding different carbon allotropes, very important from a technological point of view. In particular, fullerenes can be obtained from graphene with the use of pentagons that produce curved surfaces to be considered as wrapped-up graphene. Carbon nanotubes [50, 51] are obtained by rolling graphene along a given direction and reconnecting the carbon bonds.

Finally, since graphene, being composed of carbon, is intrinsically biocompatible, it is expected to be suitable for some biological applications.

At the end of this part of the workshop our students should have become aware of the fact that graphene is a unique crystal in the sense that it combines many superior properties, from the mechanical to the electronic viewpoint, that could be exploited in various forthcoming technological applications [52].

\section{Conclusion}

We have presented and discussed a possible workshop activity designed for students of a Master's degree in physics/electronic engineering, focused on the unique and exceptional properties characterizing the behaviour of electrons in graphene. At the end of the workshop, the students should be acquainted with the basic idea that graphene is a remarkable material from the electronic point of view and, since its various properties are different from those found in usual metals and semiconductors, it is versatile for a large number of technological applications not possible in conventional materials.

In particular, the first part of the learning activity aims to deepen student understanding about fundamental concepts of advanced physics, such as the symmetry properties of the hexagonal lattice, group theory and their application to the constructions of symmetrized wave functions in the free electron model. Great emphasis has been placed on the knowledge 
and application of group theory, stressing the fact that it allows us to predict the nature of the matrix elements of the Hamiltonian without solving the complete secular determinant. The symmetry-related aspects assume relevant importance when the crystal potential is included in the Hamiltonian. In this case, some degeneracies are removed, others may appear. It follows that the dispersion curves are appreciably deformed and affect various crystal properties such as electric, thermal, magnetic, etc.

The second part of the workshop deals with the discussion of some exotic properties, such as the behaviour of the electrons near the K-point, Klein paradox, quantum Hall effect, etc, and the technological applications of graphene, such as in solar cells, photodetectors, lasers, LEDs, chemical sensors, and so on. The quantum Hall effect in graphene, and the Cooper pairs in superconductors represent two of the very few quantum effects observable at the macroscopic level. In both cases, the correlated behaviour of the particles gives rise to effects not observable when we are dealing with processes typical of independent or weakly interacting particles, such as free electrons in metals or nearly free electrons in semiconductors. The training path also allows us to compare/differentiate the physical origin of apparently different concepts such as those of the effective mass (different from free electron mass) with that of the fractional charge and to be aware of the motivations why the interactions with the lattice affect the electron mass, whereas those with the magnetic field in a massless electron gas affect the electron charge.

The workshop, presented here, attempts to bind together concepts, such as those illustrated above, that from a superficial viewpoint may seem to be unrelated. It should be very useful to physics/engineering students because it guides them to expand their theoretical knowledge about graphene and to tackle similar problems regarding new layered materials.

Our educational environment, focused on an active engagement and application of the concepts previously introduced as separate pieces of knowledge in conjunction with the traditional lecture setting, could help students manage the physical properties of new materials more effectively and to provide them with a valuable experience useful for their future instruction. This learning path represents a viable example of integration between a theoretical teaching approach and effective learning strategies aiming to provide physics/electronic engineering students with the opportunities to apply condensed matter physics concepts and to be able to manage relevant experimental and technological aspects of graphene and other new 2D materials.

To conclude, we stress the fact that graphene is not the only stable $2 \mathrm{D}$ material of technological importance. By exploiting other layered crystals, such as silicene, stanene, germanene, boron nitride and molybdenum disulphide, it will be possible to create new 2Dbased heterostructures, and reveal and explore interesting new physical features useful to realize new ultrathin devices.

\section{Acknowledgments}

We gratefully acknowledge Professor Marisa Michelini, President of GIREP, for her invaluable support and encouragement. This work was partially supported by MIUR.

\section{Appendix A. Basic concepts of group theory}

Group theory is a powerful theoretical tool to determine the features of wave functions and to evaluate eventual degeneracies of eigenvalues. The symmetry of a body determines the ensemble of transformations that leave it unaltered. Each of them consists of one or more of 
the fundamental transformations: translation of a given quantity or its multiples, rotation through a definite angle about some axis, reflection with respect to some plane and inversion (present only in some groups). A rotation through an angle $2 \pi / n$ ( $n$ is an integer) about an axis of symmetry and a reflection plane are denoted by $\mathrm{C}_{n}$, and $\sigma$, respectively. The full set of transformations constitutes the symmetry group of a certain crystal structure. They are denoted elements of the group, and indicated with A, B, C, etc. The number of elements is called order (indicated by the symbol $h$ ). The following conditions must be satisfied:

(1) The product of two or more elements of a group (that is the successive application of two or more operations) or the square of an element is also an element of that group. The associative law is valid $((\mathrm{AB}) \mathrm{C}=\mathrm{A}(\mathrm{BC}))$, but does not necessarily have to be the commutative law, i.e. it could be $\mathrm{AB} \neq \mathrm{BA}$.

(2) Each group must contain the element $\mathrm{E}$ (called the unit element or identity), that commutes with the other elements of the group and leaves them unchanged: $\mathrm{EA}=\mathrm{AE}=\mathrm{A}$.

(3) Each element $\mathrm{A}$ has a reciprocal element $\mathrm{A}^{-1}$ (the inverse transformation), such that $\mathrm{AA}^{-1}=\mathrm{E}$. The reciprocal of the product of two or more elements is equal to the product of the reciprocals in inverse order, i.e. $(\mathrm{ABC} \ldots)^{-1}=\ldots \mathrm{C}^{-1} \mathrm{~B}^{-1} \mathrm{~A}^{-1}$.

(4) If we can isolate from a group $\mathrm{G}$ a set of elements $\mathrm{g}$ that constitute a group, then this group is called a subgroup of G. Its order $g$ is an integer factor of $h$.

(5) Two elements of a group A and B are said conjugate if there exists at least an element $\mathrm{X}$ of the group that satisfies the relation $\mathrm{A}=\mathrm{XBX}^{-1}$. The converse relation $\mathrm{B}=\mathrm{X}^{-1} \mathrm{AX}$ is also valid. If $X=E$ then $B=A$, that implies that each element is conjugate with itself. An important property of conjugate elements is that, if $\mathrm{A}$ is conjugate to $\mathrm{B}$, and $\mathrm{B}$ to $\mathrm{C}$, then $\mathrm{A}$ is conjugate to $\mathrm{C}$. The sets of conjugate elements of a group are called classes of the group. The order of a class is an integer factor of the order of the group.

The set of rotations, reflections and any combination of them that leave at least one point unchanged, constitutes a point group. We will deal with groups of this type.

From a formal point of view, we define a representation of a group as a set of mathematical operators that combine among themselves according to the rules valid for the operations of the group; they represent the group. It is customary to choose square matrices [16]. By applying a similarity transformation that scrambles rows and columns, it is possible to reduce a matrix repenting all the elements of a group to the block form. The matrices constituting the various blocks are called irreducible representations and are indicated by the symbol $\Gamma$. The order of each matrix gives the dimensionality of that representation. For most physical applications, it is sufficient to know the sum of the diagonal elements (trace) of each representation, called the character $(\chi)$.

Let us choose a generic operation $R$ of a group and indicate with $\Gamma_{i}(R)_{m n}$ the matrix element that corresponds to the row $\mathrm{m}$ and column $\mathrm{n}$ of the corresponding matrix in the $i$ th irreducible representation $i$ and with $l_{i}$ its dimensionality. This [16] implies that any irreducible representation is 'orthogonal' to the other ones, according to the expression

$$
\sum_{R} \Gamma_{i}(R)_{m n} \Gamma_{j}(R)_{m^{\prime} n^{\prime}}=\frac{\mathrm{h}}{\sqrt{1_{\mathrm{i}} \mathrm{l}_{\mathrm{j}}}} \delta_{i j} \delta_{m m^{\prime}} \delta_{n n^{\prime}}
$$

Explicitly:

If $\mathrm{i}=\mathrm{j}, \mathrm{m}=\mathrm{m}^{\prime}$ and $\mathrm{n}=\mathrm{n}^{\prime}$ the sum will give $\mathrm{h} / \mathrm{l}_{\mathrm{i}}$

If $\mathrm{m} \neq \mathrm{m}^{\prime}$ or $\mathrm{n} \neq \mathrm{n}^{\prime}$ or $\mathrm{I} \neq \mathrm{j}$, the sum will give 0 . 
Moreover, the following five properties are valid:

(1) The sum of the squares of the dimensionality $\left(l_{i}\right)$ of the matrices of all irreducible representations is equal to the order of the group: $\sum \mathrm{l}_{\mathrm{i}}^{2}=\mathrm{h}$.

(2) The sum of the squares of the characters of all irreducible representations is equal to $\mathrm{h}$ : $\sum_{\mathrm{R}}\left[\chi_{\mathrm{i}}(\mathrm{R})\right]^{2}=\mathrm{h}$.

(3) The vectors whose components are the characters of two different representations are orthogonal:

$$
\sum_{R}\left[\chi_{i}(R)\right]\left[\chi_{j}(R)\right]=0 \quad \text { if } i \neq j .
$$

(4) If the dimensionality of the matrix is greater than one, the diagonal elements are the same for all operations belonging to the same class.

(5) The number of different irreducible representations of a group is equal to the number of the classes of operations in the group.

From these properties it follows that in a crystal structure a point group has a finite number of both operations and irreducible representations. An appropriate manner for generating the basis functions for an irreducible representation, provided that its matrix elements are known, consists of applying the projection operator ${ }^{3} \mathrm{P}_{\Gamma j \mathrm{~d}}$ [17] to a generic function $\psi$ (considered as a combination/product of various simple functions such as $\mathrm{x}, \mathrm{y}, \mathrm{xy}, \mathrm{x}^{2}+\mathrm{y}^{2}$ and so on). Its definition is

$$
P_{\Gamma j d}=\sum_{R i} \chi_{j d, R i} R_{i}
$$

where $\Gamma_{\mathrm{jd}}, \mathrm{d}$ and $\chi_{\Gamma \mathrm{jd}, \mathrm{Ri}}$ indicate a representation, a diagonal element and the character, respectively. If the function $\psi$ contains terms that are bases for the selected representation, these will persist in the result, otherwise they will vanish. To simplify the calculations of the expressions and the reading of the results, bases of the lowest order are habitually chosen.

\section{Appendix B. Calculation of the lower electron energy states in monolayer graphene}

Consider, as a first example, the centre of the Brillouin zone. The $\Gamma$ point is important since almost all high symmetry lines of interest have this point as an extreme. The symmetry group is $\mathrm{C}_{6 \mathrm{v}}$, since all the operations of the symmetry group transform $\mathbf{k}$ into itself rather than into a distinct one [17]. The lowest energy point corresponds to $\mathbf{K}=0\left(\mathrm{k}_{1}=\mathrm{k}_{2}=\mathrm{h}_{1}=\mathrm{h}_{2}=0\right)$, the wave function is a constant and, consequently, it is a basis for the first irreducible representation of $\mathrm{C}_{6 \mathrm{v}}$, denoted $\Gamma_{1}$.

Higher energy states in which either $\mathrm{h}_{1}$ or $\mathrm{h}_{2}$ or both are not zero require more complex calculations. The next four higher states are $\mathrm{h}_{1}= \pm 1 ; \mathrm{h}_{2}=0$ or $\mathrm{h}_{1}=0 ; \mathrm{h}_{2}= \pm 1$. We analyze in detail the state in which $\mathrm{h}_{1}=1, \mathrm{~h}_{2}=0$ and use table 1 to derive the symmetry combinations of the degenerate functions relative to this state:

$$
\psi(\mathbf{r})=\mathrm{e}^{2 \pi \mathrm{i}[1 \xi+0 \eta]}=\mathrm{e}^{2 \pi \mathrm{i} \xi}
$$

The result of the application of these symmetry operations is shown in table B1. Inspection of the table shows that: (i) only six functions are linearly independent (those in the first six

\footnotetext{
3 This definition applies to $1 \mathrm{D}$ matrices or to the diagonal matrix elements if the dimensionality of the matrix is
} greater than one. 
Table B1. $\mathrm{C}_{6 \mathrm{~V}}$ symmetry operation effects on $\psi(\mathbf{r})$ at the $\Gamma$-point, if $\mathrm{h}_{1}=1, \mathrm{~h}_{2}=0$.

$$
\begin{aligned}
& \mathrm{E} \psi[\mathbf{r}]=\exp \left\{2 \pi \mathrm{i}\left[\mathrm{h}_{1} \xi\right]\right\}=\sigma_{\mathrm{v} 1} \\
& \mathrm{C}_{6} \psi[\mathbf{r}]=\exp \left\{2 \pi \mathrm{i}\left[\mathrm{h}_{1}(\xi-\eta)\right]\right\}=\sigma_{\mathrm{d}-2} \\
& \mathrm{C}_{-6} \psi[\mathbf{r}]=\exp \left\{2 \pi \mathrm{i}\left[\mathrm{h}_{1} \eta\right]\right\}=\sigma_{\mathrm{d} 2} \\
& \mathrm{C}_{3} \psi[\mathbf{r}]=\exp \left\{2 \pi \mathrm{i}\left[-\mathrm{h}_{1} \eta\right]\right\}=\sigma_{\mathrm{v}-2} \\
& \mathrm{C}_{-3} \psi[\mathbf{r}]=\exp \left\{2 \pi \mathrm{i}\left[\mathrm{h}_{1}(-\xi+\eta)\right]\right\}=\sigma_{\mathrm{v} 2} \\
& \mathrm{C}_{2} \psi[\mathbf{r}]=\exp \left\{2 \pi \mathrm{i}\left[-\mathrm{h}_{1} \xi\right]\right\}=\sigma_{\mathrm{d} 1} \\
& \left.\sigma_{\mathrm{v} 1} \psi[\mathbf{r}]=\exp \left\{2 \pi \mathrm{i}\left[\mathrm{h}_{1} \xi\right)\right]\right\} \\
& \sigma_{\mathrm{d} 2} \psi[\mathbf{r}]=\exp \left\{2 \pi \mathrm{i}\left[\mathrm{h}_{1} \eta\right]\right\} \\
& \sigma_{\mathrm{d}-2} \psi[\mathbf{r}]=\exp \left\{2 \pi \mathrm{i}\left[\mathrm{h}_{1}(\xi-\eta)\right]\right\} \\
& \sigma_{\mathrm{v} 2} \psi[\mathbf{r}]=\exp \left\{2 \pi \mathrm{i}\left[\mathrm{h}_{1}(-\xi+\eta)\right]\right\} \\
& \sigma_{\mathrm{v}-2} \psi[\mathbf{r}]=\exp \left\{2 \pi \mathrm{i}\left[-\mathrm{h}_{1} \eta\right]\right\} \\
& \sigma_{\mathrm{d} 1} \psi[\mathbf{r}]=\exp \left\{2 \pi \mathrm{i}\left[-\mathrm{h}_{1} \xi\right]\right\}
\end{aligned}
$$

Table B2. Character table and some simple basis functions of the irreducible representations constituting the group $\mathrm{C}_{6 \mathrm{~V}}[16,53]$.

\begin{tabular}{lrrrrrrl}
\hline $\mathbf{C}_{6 \mathrm{v}}$ & $\mathrm{E}$ & $2 \mathrm{C}_{6}$ & $2 \mathrm{C}_{3}$ & $\mathrm{C}_{2}$ & $3 \sigma_{\mathrm{d}}$ & $3 \sigma_{\mathrm{v}}$ & Bases \\
\hline$\Gamma_{1}$ & 1 & 1 & 1 & 1 & 1 & 1 & $\mathrm{z}, \mathrm{x}^{2}+\mathrm{y}^{2}, \mathrm{z}^{2}$ \\
$\Gamma_{2}$ & 1 & 1 & 1 & 1 & -1 & -1 & \\
$\Gamma_{3}$ & 1 & -1 & 1 & -1 & -1 & 1 & $\mathrm{x}^{3}-3 \mathrm{xy}^{2}$ \\
$\Gamma_{4}$ & 1 & -1 & 1 & -1 & 1 & -1 & $\mathrm{y}^{3}-3 \mathrm{yx}^{2}$ \\
$\Gamma_{5}$ & 2 & 1 & -1 & -2 & 0 & 0 & $(\mathrm{x}, \mathrm{y})$ \\
$\Gamma_{6}$ & 2 & -1 & -1 & 2 & 0 & 0 & $\left(\mathrm{x}^{2}-\mathrm{y}^{2}, \mathrm{xy}\right)$ \\
\hline
\end{tabular}

lines); (ii) each wave function has its complex conjugate. For example, the result of $\mathrm{C}_{6}$ is the complex conjugate of $\mathrm{C}_{-3}$.

A simple method for determining the symmetrized wave functions that are not null consists of inspecting the characters of two equal functions for a given irreducible representation. If they are equal, the two functions add together, if they are opposite their contributions cancel out. The formal method consisting of the application of the projection operator will give the same result. It becomes compulsory when more complex groups and higher order irreducible representations occur. Table B2 reports the characters and simple bases of the six irreducible representations constituting the group $\mathrm{C}_{6 \mathrm{~V}}$.

We first consider the irreducible representation $\Gamma_{1}$. Since the characters reported in table B2 are all 1, the application of the projection operator belonging to this representation gives the symmetrized function:

$$
\psi(\mathbf{r})_{\Gamma 1}=4\left\{\cos 2 \pi \mathrm{i}\left[\mathrm{h}_{1} \xi\right]+\cos 2 \pi \mathrm{i}\left[\mathrm{h}_{1} \eta\right]+\cos \left\{2 \pi \mathrm{i}\left[\mathrm{h}_{1}(\xi-\eta)\right]\right\}\right.
$$

that is a basis for $\Gamma_{1}$ since it behaves similarly to $x^{2}+y^{2}$.

When we consider the next irreducible representation $\Gamma_{2}$ we note that all operations that give the same wave function have opposite characters. Consequently, the first six functions cancel out with the remaining ones and there is no symmetrized combination belonging to $\Gamma_{2}$. A similar result is found when we select the representation $\Gamma_{4}$. For the other $1 \mathrm{D}$ representation $\Gamma_{3}$ all equal terms have the same sign, but the complex conjugate of a couple of functions has opposite sign with respect to the original one. Consequently, only imaginary terms appear in the symmetrized contribution to the representation 


$$
\psi(\mathbf{r})_{3}=4 \mathrm{i}\left\{\sin 2 \pi \mathrm{i}\left[\mathrm{h}_{1} \xi\right]+\sin 2 \pi \mathrm{i}\left[\mathrm{h}_{1} \eta\right]+\sin \left\{2 \pi \mathrm{i}\left[\mathrm{h}_{1}(\xi-\eta)\right]\right\} .\right.
$$

The computation is more complex for the $2 \mathrm{D}$ representations, $\Gamma 5$ and $\Gamma_{6}$. In this case we have two symmetrized combinations for each representation that are the result of the application of the two projection operators: the first combination transforms as the first basis, the second as the second basis. The set of symmetry operators corresponding to the reflection planes does not contribute since the characters are zero. The calculation of the symmetrized combination can be carried out according to the guidelines illustrated above, with the difference that the extended character table (reported in various textbooks [16-18, 53]) must be used. Similar results are obtained when the remaining three wave functions are selected.

The lines $T_{i}$ join the point $\Gamma$ with the points $K_{i}(i=0, \ldots, 5)$; the first one, $T_{0}$, joins $\Gamma$ with $K_{0}$, whose coordinates are $\left[1 / 3 \mathbf{b}_{1}, 1 / 3 \mathbf{b}_{2}\right]$, located at a reciprocal distance $2 /$ (3a) from $\Gamma$. A wave propagating along $T_{0}$ is characterized by $k_{1}=k_{2}$ and its function is

$$
\psi_{K}(\mathbf{r})_{T 0}=\psi_{k h}(\mathbf{r})=\mathrm{e}^{2 \pi \mathrm{i}\left[\left(\mathrm{k}_{1}+\mathrm{h}_{1}\right) \cdot \xi+\left(\mathrm{k}_{1}+\mathrm{h}_{2}\right) \eta\right]} .
$$

In the lowest energy level, both $\mathrm{h}_{1}$ and $\mathrm{h}_{2}$ are zero and the function reduces to

$$
\psi_{K}(\mathbf{r})_{T 0}=\psi_{k h}(\mathbf{r})=\mathrm{e}^{2 \pi \mathrm{i}\left[\mathrm{k}_{1} \cdot(\xi+\eta)\right]} .
$$

The application of the symmetry operations of the $\mathrm{C}_{6 \mathrm{v}}$ group gives some redundant results. In fact, only two functions are physically different, they are $\exp \left\{2 \pi \mathrm{i}\left[\mathrm{k}_{1}(\xi+\eta)\right]\right\}$ and $\exp \left\{2 \pi \mathrm{i}\left[\mathrm{k}_{1}(\xi-\eta)\right]\right\}$, the others are their complex conjugate. It follows that the symmetry group is the subgroup $\mathrm{C}_{2 \mathrm{v}}$ of $\mathrm{C}_{6 \mathrm{v}}$; it consists of the symmetry operations $\mathrm{E}, \mathrm{C}_{2} \sigma_{\mathrm{v}}(\mathrm{xz})$ and $\sigma_{\mathrm{v}}(\mathrm{yz})$. Using the table of characters reported in several textbooks [16-18, 53] and following the procedure adopted for the $\Gamma$ point, it is straightforward to deduce the symmetrized combinations. Analogous reasoning can be carried out for other values or combinations of $h_{1}$ and $\mathrm{h}_{2}$.

Finally, we consider the symmetry properties at the K-points. The coordinates of the first three are $K_{0}=\left[1 / 3 \mathbf{b}_{1}, 1 / 3 \mathbf{b}_{2}\right], K_{1}=\left[-1 / 3 \mathbf{b}_{1}, 2 / 3 \mathbf{b}_{2}\right]$ and $K_{2}=\left[2 / 3 \mathbf{b}_{1},-1 / 3 \mathbf{b}_{2}\right]$. The others $\mathrm{K}_{3}, \mathrm{~K}_{-1}, \mathrm{~K}_{-2}$ are symmetric with respect to $\mathrm{K}_{0}, \mathrm{~K}_{1}$ and $\mathrm{K}_{2}$, respectively. They can be obtained by adding/subtracting a reciprocal lattice vector to those of the first set; consequently, they are not noteworthy. The points $\mathrm{K}_{1}$ and $\mathrm{K}_{2}$ can be obtained from $\mathrm{K}_{0}$ through the rotations $\mathrm{C}_{6}$ and $\mathrm{C}_{-6}$, respectively, $\mathrm{K}_{3}, \mathrm{~K}_{-1}, \mathrm{~K}_{-2}$ through the rotations $\mathrm{C}_{2}, \mathrm{C}_{3}$ and $\mathrm{C}_{-3}$, respectively. The vertical reflection planes $3 \sigma_{\mathrm{d}}$ and $3 \sigma_{\mathrm{v}}$ produce the same results, consequently the symmetry group is $\mathrm{C}_{6 \mathrm{v}}$ and the same considerations discussed for the $\Gamma$ point apply [17].

\section{ORCID iDs}

Dominique Persano Adorno (ib https://orcid.org/0000-0001-7655-1114

\section{References}

[1] Le Lay G, Salomon E and Angot T 2016 Silicene: silicon conquers the 2D world Europhys. News 47 17-21

[2] Mermin N D and Wagner H 1966 Absence of ferromagnetism or antiferromagnetism in one- or two-dimensional isotropic heisenberg models Phys. Rev. Lett. $171133-6$

[3] Ajayan P, Kim P and Banerjee K 2016 Two-dimensional van der Waals materials Phys. Today 69 $38-44$

[4] Novoselov K S et al 2004 Electric field effect in atomically thin carbon films Science 306 666-9

[5] Geim A K and Novoselov K S 2007 The rise of graphene Nat. Mater. 6 183-91 
[6] Castro Neto A H, Guinea F, Peres N M R, Novoselov K S and Geim A K 2009 The electronic properties of graphene Rev. Mod. Phys. 81 109-61

[7] Malard L M, Guimarães M H D, Mafra D L, Mazzoni M S C and Jorio A 2009 Group-theory analysis of electrons and phonons in N-layer graphene systems Phys. Rev. B 79 125426-8

[8] Kogan E and Nazarov V U 2012 Symmetry classification of energy bands in graphene Phys. Rev. B 85 115418-5

[9] Wilson M 2006 Electrons in atomically thin carbon sheets behave like massless particles Phys. Today 59 21-3

[10] Novoselov K S, Falko V I, Colombo L, Gellert P R, Schwab M G and Kim K 2012 A roadmap for graphene Nature 490 192-200

[11] http://graphene-flagship.eu/project

[12] Persano Adorno D, Pizzolato N and Fazio C 2015 Elucidating the electron transport in semiconductors via Monte Carlo simulations: an inquiry-driven learning path for engineering undergraduates Eur. J. Phys. 36055017

[13] Persano Adorno D and Pizzolato N 2015 An inquiry-based approach to the Franck-Hertz experiment Il Nuovo Cimento C 38109

[14] Pizzolato N, Fazio C, Sperandeo Mineo R M and Persano Adorno D 2014 Open-inquiry driven overcoming of epistemological difficulties in engineering undergraduates: a case study in the context of thermal science Phys. Rev. ST 10010107

[15] Bouckaert L P, Smoluchowski R and Wigner E 1936 Theory of Brillouin zones and symmetry properties of wave functions in crystals Phys. Rev. 50 58-67

[16] Tinkham M 1964 Group Theory and Quantum Mechanics (New York: McGraw-Hill)

[17] Slater 1965 Quantum Theory of Molecules and Solids vol 2 (New York, USA: McGraw-Hill)

[18] Nussbaum A 1966 Crystal symmetry, group theory, and band structure calculations Solid State Physics-Advances in Research and Applications ed F Seitz and D Turnbull (New York: Academic Press) pp 165-272

[19] Huang H, Zheng F, Zhang P, Wu J, Gu B L and Duan W 2014 A general group theoretical method to unfold band structures and its application New J. Phys. 16033034

[20] Ashcroft N W and Mermin N D 1976 Solid State Physics (Orlando, FL: Saunders College Publishing)

[21] Wallace P R 1947 The band theory of graphite Phys. Rev. 71 622-34

[22] Kittel C 2005 Introduction to Solid State Physics 8th Edn (New York: Wiley)

[23] Kittel C 1987 Quantum Theory of Solids 2nd Revised Edn (New York: Wiley)

[24] Lomer W M 1955 The valence bands in two-dimensional graphite Proc. R. Soc. Lon. A 227 330-49

[25] Kogan E, Nazarov V U, Silkin V M and Kaveh M 2014 Energy bands in graphene: comparison between the tight-binding model and ab initio calculations Phys. Rev. B 89165430

[26] Kogan E and Silkin V M 2017 Electronic structure of graphene: (nearly) free electrons bands vs. tight-binding bands Phys. Status Solidi B 2541700035

[27] Cooper D R et al 2012 Experimental review of graphene ISRN Condensed Matter Physics 501686 $1-56$

[28] Katsnelson M I, Novoselov K S and Geim A K 2006 Chiral tunnelling and the Klein paradox in graphene Nat. Phys. 2 620-5

[29] Robinson T R 2012 On Klein tunneling in graphene Am. J. Phys. 80 141-7

[30] Torres-Silva H and Torres Cabezas D 2012 Chiral waves in graphene tunneling IJRRAS 12 6-11

[31] Dombey N and Calogeracos A 1999 Seventy years of the Klein paradox Phys. Rep. 315 41-59

[32] Stormer H L 1999 Nobel lecture: the fractional quantum Hall effect Rev. Mod. Phys. 71 875-89

[33] Longo M 2011 Effetto Hall su graphene PhD Thesis Università degli Studi della Calabria

[34] Tsui D C, Stormer H L and Gossard A C 1982 Two-dimensional magnetotransport in the extreme quantum limit Phys. Rev. Lett. 48 1559-62

Tsui D C 1999 Nobel Lecture: Interplay of disorder and interaction in two-dimensional electron gas in intense magnetic fields Rev. Mod. Phys. 71 891-5

[35] Laughlin R B 1983 Anomalous quantum Hall effect: an incompressible quantum fluid with fractionally charged excitations Phys. Rev. Lett. 501395

[36] Solyom J 2014 Wigner crystals: new realizations of an old idea EPJ Web of Conference 7801009

[37] Novoselov K S, Geim A K, Morozov S V, Jiang D, Katsnelson M I, Grigorieva I V, Dubonos S V and Firsov A A 2005 Two-dimensional gas of massless Dirac fermions in graphene Nature 438 197-200 
[38] Borghi G 2007 Correlazioni elettroniche e modi collettivi nel grafene PhD Thesis Università degli Studi di Pisa

[39] Novoselov K S, Jiang Z, Zhang Y, Morozov S V, Stormer H L, Zeitler U, Maan J C, Boebinger G S, Kim P and Geim A K 2007 Room-temperature quantum Hall effect in graphene Science 3151379

[40] Johnson B L and Kirczenow G 1997 Composite fermions in the quantum Hall effect Rep. Prog. Phys. 60889

Jain J K 2000 The composite fermion: a quantum particle and its quantum fluids Physics Today 4 $39-45$

[41] Li X and Zhu H 2016 The graphene-semiconductor Schottky junction Phys. Today 69 46-51

[42] Lee P A and Ramakrishnan T V 1985 Disordered electronic systems Rev. Mod. Phys. 57287

[43] Tworzydlo J, Snyman I, Akhmerov A R and Beenakker C W J 2007 Valley-isospin dependence of the quantum Hall effect in a graphene p-n junction Phys. Rev. B 76035411

[44] Rycerz A, Tworzydlo J and Beenakker C W J 2007 Valley filter and valley valve in graphene Nat. Phys. 3 172-5

[45] Spezia S, Persano Adorno D, Pizzolato N and Spagnolo B 2010 Temperature dependence of spin depolarization of drifting electrons in n-type GaAs bulks Acta Phys. Pol. B 41 1171-80 http: / /actaphys.uj.edu.pl/fulltext?series $=$ Reg\&vol $=41 \&$ page $=1171$

[46] Spezia S, Persano Adorno D, Pizzolato N and Spagnolo B 2010 Relaxation of electron spin during high-field transport in GaAs bulk J. Stat. Mech. P11033

[47] Spezia S, Persano Adorno D, Pizzolato N and Spagnolo B 2012 New insight in electron spin dynamics in the presence of correlated noise J. Phys.: Condens. Matter 24052204

Spezia S, Persano Adorno D, Pizzolato N and Spagnolo B 2013 Enhancement of electron spin lifetime in GaAs crystals: the benefits of dichotomous noise EPL 10447011

[48] Blau S K 2017 Conduction electrons flow like honey Phys. Today (https://doi.org/10.1063/ PT.6.1.20170907a)

[49] Kumar K et al 2017 Superballistic flow of viscous electron fluid through graphene constrictions Nat. Phys. (https://doi.org/10.1038/nphys4240)

[50] Saito R, Dresselhaus G and Dresselhaus M S 1998 Physical Properties of Carbon Nanotubes (London: Imperial College Press)

[51] Charlier J C, Blase X and Roche S 2007 Electronic and transport properties of nanotubes Rev. Mod. Phys. 79677

[52] Novoselov K S 2011 Nobel lecture: graphene: materials in the flatland Rev. Mod. Phys. 83 837-49

[53] Koster G F and Lomont J S 1957 Applications of finite groups Solid State Physics vol 5 (New York: Academic Press) 